\title{
ORIGEN DEL POLEN DE ARTEMISIA EN LA ATMÓSFERA DE EXTREMADURA (SO ESPAÑA)
}

\author{
Inmaculada SILVA, Rafael TORMO, Adolfo F. MUÑOZ, \\ Juana TAVIRA y Alfonsa MORENO
}

\begin{abstract}
RESUMEN. Origen del polen de Artemisia en la atmósfera de Extremadura (SO España). Mediante tres captadores volumétricos de tipo Hirst se ha podido conocer la concentración de granos de polen de Artemisia en Extremadura entre 1993 y 1998 para Badajoz y entre 1996 y 1998 para Cáceres y Mérida. Su presencia es muy esporádica y la cantidad reducida, aparece centrada fundamentalmente entre agosto y noviembre, aunque se detectan también en menor cantidad entre enero y marzo, la cantidad encontrada nunca llega a los 15 granos por metro cúbico de media diaria y la suma total de las concentraciones diarias es algo inferior a 40. Dado que apenas existe representación de especies del género Artemisia se discute su transporte desde la parte oriental de la península ibérica donde su concentración es mucho más importante, esta hipótesis es apoyada por los resultados de las correlaciones con los vientos procedentes del este y la fenología de las especies presentes en dichas áreas.
\end{abstract}

Palabras clave. Aerobiología, polen, Asteraceae, Artemisia, Extremadura, meteorología, alergia.

ABSTRACT. Source of Artemisia pollen in the Extremadura atmosphere (SW Spain). Using three Hirst volumetric sporetraps Artemisia pollen concentrations in Extremadura has been measured between 1993 and 1998 in Badajoz and between 1996 and 1998 in Cáceres and Mérida. Its presence is very sporadic and the amount limited It appears mainly between August and November, although, in a lesser amount, it is present from January to March. The estimated concentrations never rises to 15 pollen grains per cubic meter of air and the daily concentration sum is lower than 40 . As there are scarcely any individual of Artemisia genus in the studied area its transport from the west of Iberian peninsula, where its distribution is much more important, is discussed. This hypothesis is supported by correlation results between westerly wind and the phenology of the species present in theses areas.

Key words. Aerobiology, pollen, Asteraceae, Artemisia, Extremadura, meteorology. allergy.

\section{INTRODUCCIÓN}

El polen de Artemisia constituye, dentro de la familia Asteraceae, uno de los tipos de mayor potencial alergénico, así, aunque la mayor parte de los estudios disponibles no son comparables, ya que las metodologías empleadas en los mismos no son homogéneas, los datos del estudio Alergológica (1995) muestran una sensibilización a Artemisia en

Trabajo realizado gracias a la ayuda concedida por la Consejería de Educación y Juventud de la Junta de Extremadura con cargo a los proyectos EIB94-12 y PR 197D048. 
España muy variable según las regiones, con cifras del 27,3\% y 24,1\% en Aragón y Murcia, respectivamente, que contrastan con las de la zona Norte, entre el 1-3\%. En Extremadura, en concreto en Badajoz, el porcentaje sensible al polen de este género es del $66 \%$ de los pacientes sospechosos de patologías alérgicas (Gonzalo et al., 1997).

A pesar de su amplia distribución y su frecuente aparición, la concentración en el aire del polen de Artemisia casi nunca alcanza niveles elevados (Spieksma \& Von Wahl, 1991), además, las concentraciones con las que aparece este tipo en la atmósfera europea son variables, comprendiendo entre el $0,5 \mathrm{y}$ el $7 \%$ del contenido total del polen atmosférico de las estaciones estudiadas por Spieksma \& Von Wahl (1991) y Frenguelli et al. (1986). En España, desde el punto de vista aeropalinológico, los enclaves donde mayor cantidad de polen se concentra en la atmósfera son las poblaciones estudiadas del este peninsular, encontrándose los máximos registrados en Chirivel (Almería) (Cariñanos et al. 1998a y b), Murcia (Munuera et al., 1995), Zaragoza (Bermejo et al., 1998a y b) y Lérida (Belmonte et al., 1998a).

En la Península Ibérica de acuerdo con Tutin et al. (1976) se encuentran las siguientes especies de Artemisia: A. verlotiorum Lamotte, A. absinthium L., A. arborescens L., A. alba Turra, A. caerulescens L., A. abrotanum L., A. campestris L., A. granatensis Boiss., A. reptans C. Sm., A. barrelieri Besser, A. herba-alba Asso, A. pedemontana Balbis, A. vulgaris L., A. chamaemelifolia Vill. y A. eriantha Ten. De éstas, algunas están restringidas a las zonas litorales y zonas montanas. En cuanto a la distribución del género en Extremadura, Devesa (1995) describe sólo la existencia de dos especies, ambas poco comunes, A. vulgaris y A. verlotiorum.

Teniendo en cuenta la alta incidencia de personas sensibles a este polen, al menos en la ciudad de Badajoz, y su escasa representación en la región, el presente artículo pretende buscar una explicación a la dinámica de variaciones de las concentraciones de Artemisia, partiendo del hecho de que ésta va a depender del transporte de granos a partir de poblaciones situadas fuera de los límites regionales.

\section{MATERIAL Y MÉTODOS}

El estudio se ha llevado a cabo mediante el seguimiento, durante los años 1993 a 1998, de las variaciones diarias y horarias de las concentraciones de granos de polen de Artemisia en la atmósfera de Badajoz, y de 1996 a 1998 en las de Mérida y Cáceres, mediante captadores volumétricos de metodología Hirst, analizándose posteriormente las muestras mediante cuatro barridos, utilizando microscopía óptica a 400 aumentos.

Para buscar las fuentes del polen registrado en Extremadura, se ha realizado un análisis de las correlaciones existentes entre las concentraciones totales mensuales encontradas en Badajoz (1995, 1996 y 1997), Mérida y Cáceres (ambas en 1996 y 1997) y los encontrados en el resto de las localidades españolas, utilizando sólo aquellas para las que se poseen datos de 1995, 1996 y 1997. Para ello, los datos se han extraído de las tablas publicadas periódicamente por los equipo integrantes de la Red Española de Aerobiología, todos los cuales utilizan la misma metodología que el presente estudio (Aira et al., 1998a y b; Belmonte et al., 1998a, b, c, d, e, f, g y h; Bermejo et al., 1998a y b; Cabezudo et al., 1998; Candau et al., 1998a y b; Cariñanos et al., 1998a y b; De Pablos et al., 1998; Díaz de la Guardia \& Alba 1998a y b; Fernández et al., 1998a y b; Galán et al., 1998a y b; González et al., 1998a y b; Gutiérrez \& Navarro, 1998; Gutiérrez et al., 1998; Iglesias et al., 1998a y b; Moreno et al., 1998a y b; Moreno-Grau et al., 1998a y b; Recio et al., 1998; Rodríguez et 
al., 1998a y b; Ruiz et al., 1998a y b; Silva et al., 1998a y b; Tavira et al., 1998a y b; Toro et al., 1998; Trigo et al., 1998; Valencia et al., 1998). Como resumen de los datos reflejados en la citada bibliografía, en la figura 1 se muestra, de forma orientativa, las concentraciones anuales registradas en 1997.

Para estudiar la incidencia de los parámetros meteorológicos sobre las concentraciones diarias del polen de Artemisia en la Región, se ha calculado el coeficiente de correlación de Spearman (r) entre los datos de concentración diaria de polen de Artemisia (en granos por metro cúbico), únicamente los datos de la estación de Badajoz, por disponer de una serie de seis años, con los datos meteorológicos diarios relativos a temperaturas medias, máximas y mínimas (en grados centígrados), humedad relativa (en \%), precipitación (en $\mathrm{mm}$ ), velocidad del viento (en $\mathrm{Km} / \mathrm{h}$ ), período de calma (horas diarias), así como los períodos de viento procedentes de los cuadrantes 1 (NE), 2 (SE), 3 ( $\mathrm{SO}$ ) y 4 (NO) (en horas diarias), que han sido proporcionados por el Centro Meteorológico Territorial de Extremadura, y proceden de la Estación Automática de Talavera la Real. Además de dicho coeficiente de

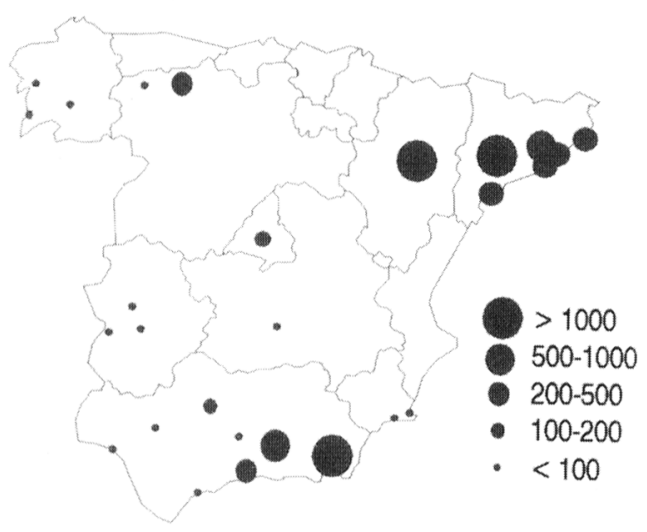

Figura 1. Representación de las concentraciones totales anuales (g.p. $/ \mathrm{m}^{3}$ ) de Artemisia registradas durante 1997 en distintas estaciones españolas de la REA.

\begin{tabular}{lccc}
\hline Estación & Badajoz & Mérida & Cáceres \\
\hline 1993 & 11,0 & & \\
1994 & 17,7 & & \\
1995 & 6,1 & & \\
1996 & 34,1 & 15,8 & 39,5 \\
1997 & 23,7 & 27,9 & 27,3 \\
1998 & 21,9 & 31,0 & 19,4 \\
\hline
\end{tabular}

Tabla 1. Valores de la suma anual de las concentraciones diarias de polen en las tres estaciones durante los años de estudio.

correlación se ha calculado la probabilidad de que $r=0(p)$.

\section{RESULTADOS}

El polen de Artemisia aparece de forma esporádica en las tres estaciones estudiadas, así, en la tabla 1 se muestran las sumas de las concentraciones diarias durante los años de estudio en las distintas estaciones, observando como en Badajoz, éstas oscilaban entre el valor de 6,1 de 1995 hasta 34,1 de 1996, en la de Mérida entre el valor de 15,8 de 1996 y 31,0 de 1998 y en Cáceres entre el valor de 19,4 de 1998 y 39,5 de 1996.

En cuanto a la distribución de las concentraciones diarias, (figs. 2 y 3 ) se observa, para la estación de Badajoz, cómo en 1993, la concentración máxima se alcanzó el 30 de agosto con 2,4 g.p. $/ \mathrm{m}^{3}$, existiendo dos períodos de aparición, uno entre finales de agosto y principios de septiembre y otro en noviembre, en 1994 las máximas concentraciones se alcanzaron el 5 de septiembre y el 1 de diciembre, ambas con 2,4 g.p. $/ \mathrm{m}^{3}$, existiendo dos períodos de polinización marcados, uno entre finales de agosto y principios de septiembre y otro entre finales de noviembre y principios de diciembre, y en 1995 la concentración máxima se alcanza el 15 de agosto con 1,8 g.p. $/ \mathrm{m}^{3}$, estando las concentraciones agrupadas en dos períodos, uno a finales de enero y el otro en agosto, aunque con apariciones en noviembre y 

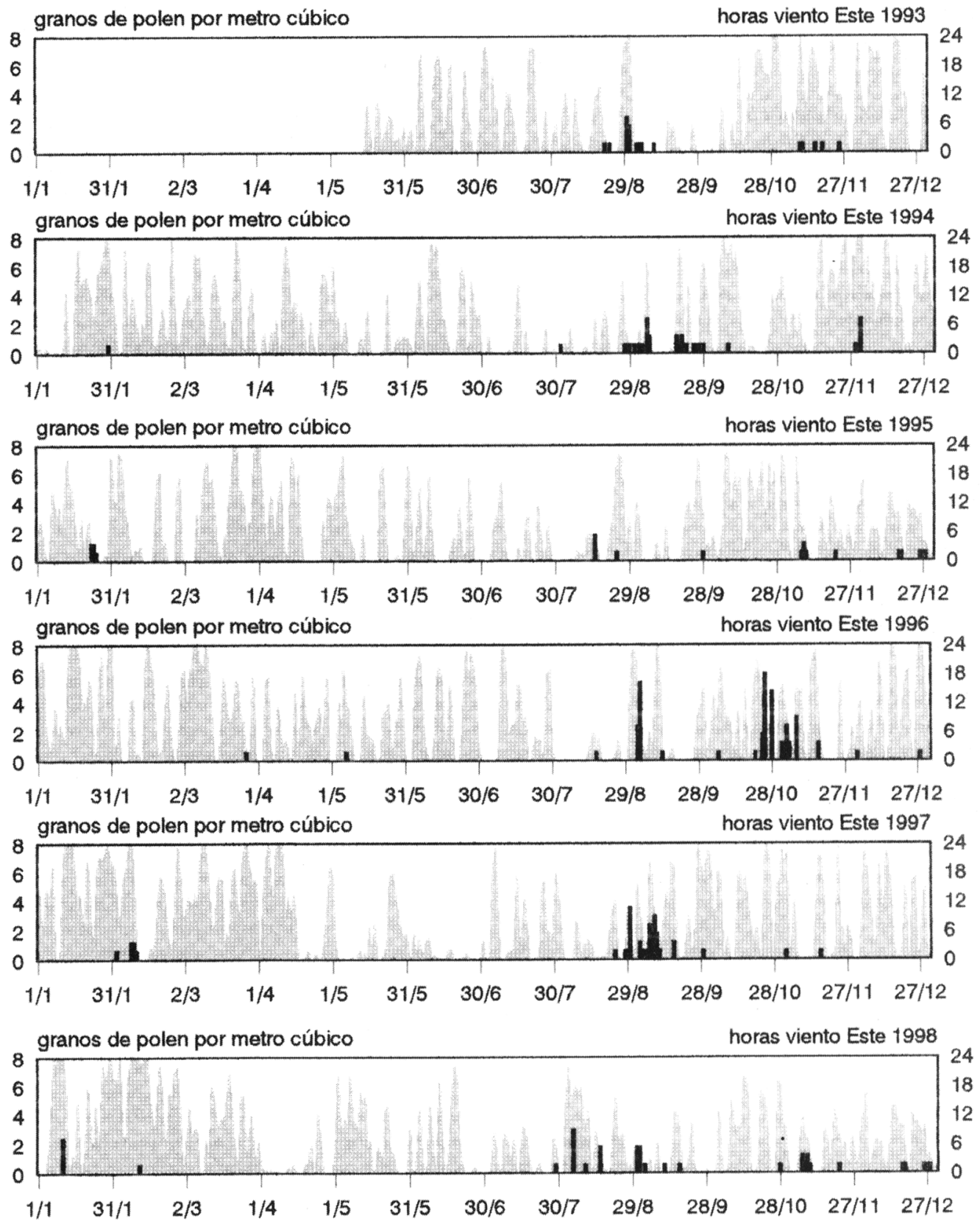

Figura 2. Concentraciones diarias de polen (en g.p. $/ \mathrm{m}^{3}$ ) de Artemisia en Badajoz, durante el período de 1993 a 1998. 

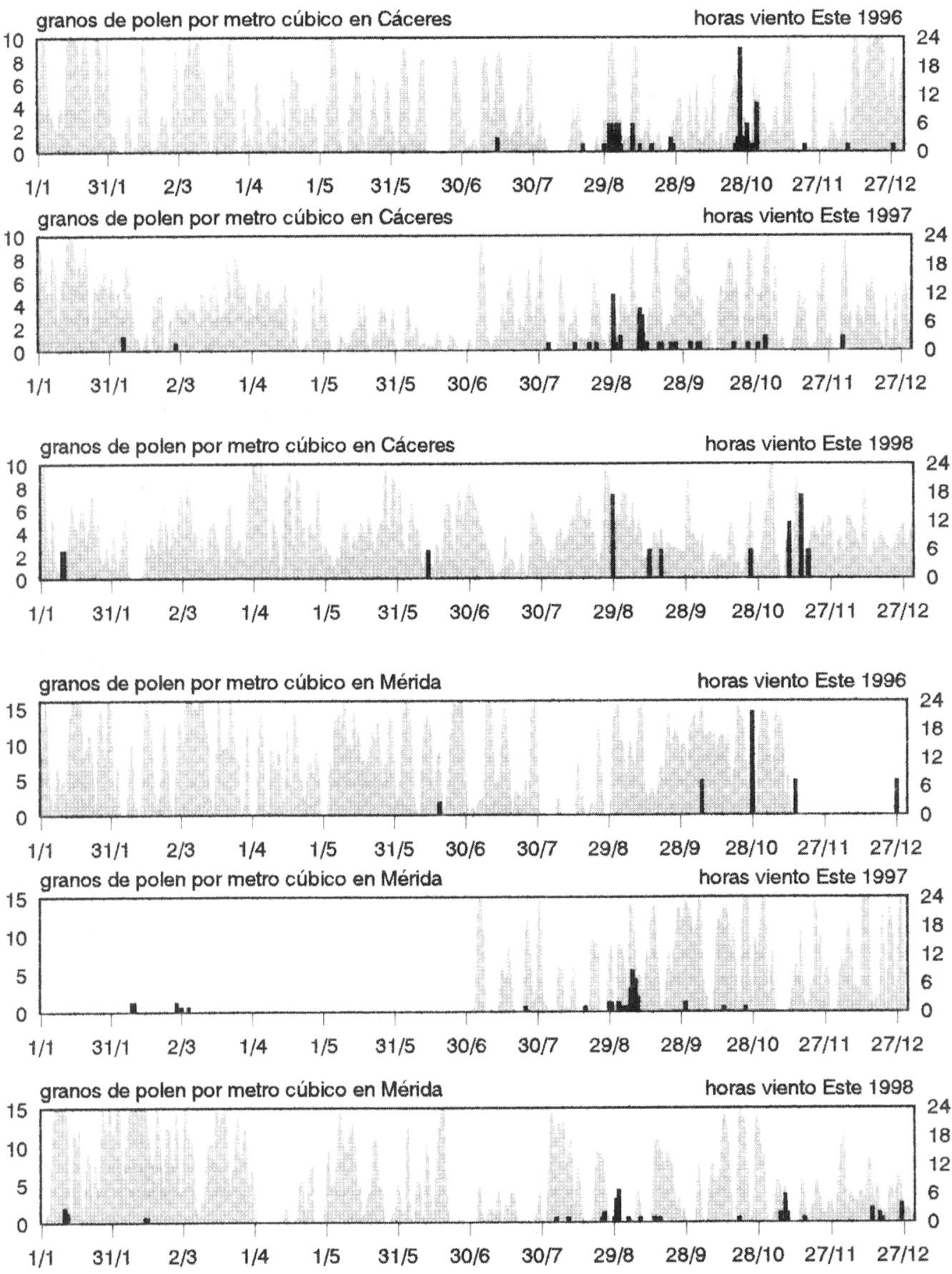

Figura 3. Concentraciones diarias de polen (en g.p. $/ \mathrm{m}^{3}$ ) de Artemisia en Cáceres y Mérida para el período 1996-1998. 
diciembre.

En 1996, Badajoz alcanza su concentración máxima el 24 de octubre con 6,1 g.p. $/ \mathrm{m}^{3}$, Cáceres el 24 de octubre con 9,1 g.p./ $\mathrm{m}^{3}$ y Mérida el 27 de octubre con 14,4 g.p. $/ \mathrm{m}^{3}$, las tres estaciones muestran dos períodos de aparición, uno entre finales de agosto y principios de septiembre y otro entre finales de octubre y principios de noviembre. En 1997, Badajoz alcanza el máximo el 30 de agosto con 3,7 g.p. $/ \mathrm{m}^{3}$, Cáceres el 30 de agosto con 4,9 g.p. $/ \mathrm{m}^{3}$ y Mérida el 8 de septiembre con 5,5 g.p. $/ \mathrm{m}^{3}$, se observa en este año una aparición centrada entre finales de agosto y principios de septiembre, con apariciones esporádicas en el otoño e invierno. En 1998, la concentración máxima en Badajoz se registra el 5 de agosto con 3,0 g.p. $/ \mathrm{m}^{3}$, en Cáceres el 29 de agosto con 7,3 g.p. $/ \mathrm{m}^{3}$ y en Mérida, el 31 de agosto con 4,3 g.p. $/ \mathrm{m}^{3}$, en este año las mayores concentraciones aparecen también entre finales de agosto y principios de septiembre, existiendo apariciones, como en el año anterior, durante el otoño y el invierno.

El estudio de las correlaciones entre los valores mensuales de las sumas de las concentraciones diarias en las estaciones extremeñas y las del resto de España, se presenta en la tabla 2, en ella se observa, en primer lugar la significación de las correlaciones encontradas entre las tres localidades extremeñas (todas ellas con $\mathrm{p}<0,05)$. En cuanto al resto, las concentraciones polínicas de los 36 meses comparados en la estación de Badajoz (1995, 1996 y 1997), mostró correlaciones significativas con todas las estaciones estudiadas. Los dos años que se comparan en el caso de Cáceres (1996 y 1997), mostraron también correlaciones con el resto de las estaciones. En el caso de las correlaciones en la ciudad de Mérida (1996 y 1997), no resultaron significativas las realizadas con los

\begin{tabular}{|c|c|c|c|c|c|c|c|c|c|c|}
\hline & & Huelva & Sevilla & Córdoba & Jaén & Estepona & Málaga & Granada & Chirivel & Cartagena \\
\hline \multirow[t]{3}{*}{ Badajoz } & $r$ & 0,4632 & 0,4762 & 0,5773 & 0,4789 & 0,6042 & 0,5724 & 0,5790 & 0,5353 & 0,4322 \\
\hline & $\mathrm{p}$ & 0,004 & 0,003 & 0,0001 & 0,003 & 0,0001 & 0,0001 & 0,0001 & 0,0001 & 0,008 \\
\hline & $\mathrm{n}$ & 36 & 36 & 36 & 36 & 36 & 36 & 36 & 32 & 36 \\
\hline \multirow[t]{3}{*}{ Mérida } & $\mathrm{r}$ & 0,4506 & 0,4239 & 0,4692 & 0,3256 & 0,4683 & 0,5025 & 0,4698 & 0,4004 & 0,1838 \\
\hline & $\mathrm{p}$ & 0,027 & 0,039 & 0,021 & 0,121 & 0,021 & 0,012 & 0,021 & 0,053 & 0,390 \\
\hline & $\mathrm{n}$ & 24 & 24 & 24 & 24 & 24 & 24 & 24 & 24 & 24 \\
\hline \multirow[t]{4}{*}{ Cáceres } & $\mathrm{r}$ & 0,5747 & 0,7147 & 0,8024 & 0,6460 & 0,7427 & 0,6861 & 0,7315 & 0,7563 & 0,4955 \\
\hline & $\mathrm{p}$ & 0,003 & 0,0001 & 0,0001 & 0,0001 & 0,0001 & 0,0001 & 0,0001 & 0,0001 & 0,014 \\
\hline & $n$ & 24 & 24 & 24 & 24 & 24 & 24 & 24 & 24 & 24 \\
\hline & & Madrid & León & Barcelona & Zaragoza & Bellaterra & & Badajoz & Mérida & \\
\hline \multirow[t]{3}{*}{ Badajoz } & $r$ & 0,5815 & 0,3659 & 0,4419 & 0,4903 & 0,5720 & & & & \\
\hline & $\mathrm{p}$ & 0,0001 & 0,028 & 0,007 & 0,002 & 0,0001 & & & & \\
\hline & $\mathrm{n}$ & 36 & 36 & 36 & 36 & 36 & & & & \\
\hline \multirow[t]{3}{*}{ Mérida } & $r$ & 0,5003 & 0,3141 & 0,3127 & 0,3924 & 0,4128 & & 0,5170 & & \\
\hline & $\mathrm{p}$ & 0,013 & 0,135 & 0,137 & 0,058 & 0,045 & & 0,010 & & \\
\hline & $\mathrm{n}$ & 24 & 24 & 24 & 24 & 24 & & 36 & & \\
\hline \multirow[t]{3}{*}{ Cáceres } & $r$ & 0,8406 & 0,5567 & 0,7435 & 0,7419 & 0,8113 & & 0,7225 & 0,5042 & \\
\hline & $\mathrm{p}$ & 0,0001 & 0,005 & 0,0001 & 0,0001 & 0,0001 & & 0,0001 & 0,012 & \\
\hline & $\mathrm{n}$ & 24 & 24 & 24 & 24 & 24 & & 36 & 36 & \\
\hline
\end{tabular}

Tabla 2. Coeficientes de correlación de Spearman (r), valor de la probablidad de $r=0$ (p) y número de meses analizados (n), calculadas entre los valores mensuales de las sumas de las concentraciones diarias encontradas de 1995 a 1997 de las estaciones extremeñas con las del resto de España (en negrita aparecen aquellas correlaciones con $\mathrm{p}<0,05)$. 


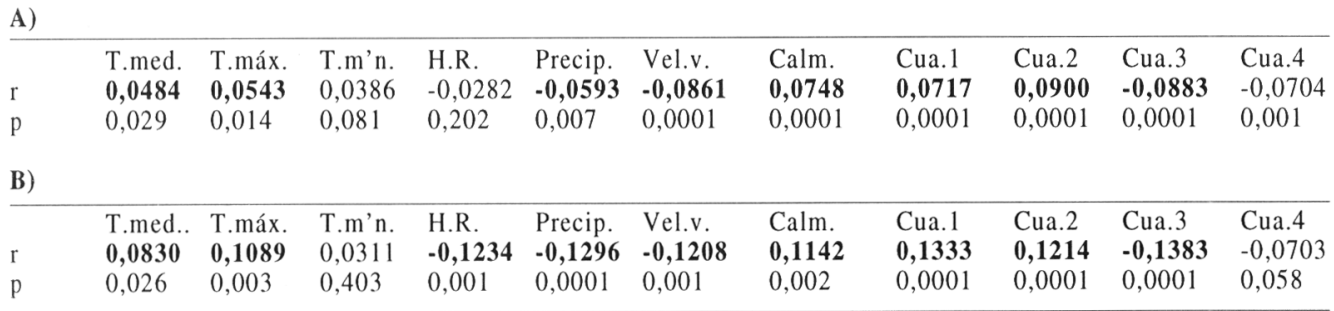

Tabla 3. Coeficientes de correlación de Spearman ( $r$ ) y probablidad de $r=0$ (p) entre las concentraciones diarias de polen de Artemisia en la ciudad de Badajoz, de 1993 a 1998, y los valores de los parámetros meteorológicos. A) usando todos los días de estudio ( $\mathrm{n}=2056$ días). B) usando sólo los meses de agosto, septiembre, octubre y noviembre ( $\mathrm{n}=732$ días), (en negrita aparecen aquellas correlaciones con $\mathrm{p}<0,05)$.

datos de Jaén, Cartagena, León, Barcelona y Zaragoza. También se han vuelto a calcular los mismos coeficientes de correlación pero utilizando sólo los meses de agosto a diciembre, apareciendo en este caso sólo significación positiva de la estación de Badajoz con las estaciones de León, Madrid, Córdoba y Sevilla; de la estación de Cáceres con las estaciones de León y Madrid; y de la estación de Mérida con la estación de Estepona.

En lo referente al estudio de las correlaciones entre las concentraciones diarias de polen de Artemisia y los valores diarios de los distintos parámetros meteorológicos registrados en la estación de Badajoz, la tabla 3 muestra cómo se encontraron correlaciones significativas con todos los parámetros $(\mathrm{P}<0,05)$, salvo con las temperaturas mínimas, con las precipitaciones y con los períodos de viento procedentes del cuadrante 4. Estas correlaciones mostraron la misma significación, cuando en el análisis se tomaron todos los días de muestreo (2056 días), y cuando sólo se utilizaron los datos relativos a los meses de agosto, septiembre, octubre y noviembre (732 días), elegidos en función de ser el período de mayor polinización. Las correlaciones fueron positivas en el caso de las temperaturas medias y máximas, en el de los períodos de calmas, y los períodos con vientos procedentes de los cuadrantes 1 y 2 (vientos del Este), y fueron negativas en el caso de la humedad, la velocidad del viento y los períodos de vientos procedentes del cuadrante 3 .

\section{DISCUSIÓN Y CONCLUSIONES}

Las concentraciones registradas son bajas en toda la Región, estando el valor de la suma anual de las concentraciones diarias siempre por debajo del valor de 40, y no existen grandes diferencias entre las tres localidades estudiadas.

Con respecto al calendario de aparición, se observa un comportamiento muy similar entre las tres localidades, coincidiendo la época en la que aparecen las concentraciones máximas diarias en cada una de ellas durante los años de estudio, este comportamiento lleva consigo la existencia de correlaciones significativas entre las tres estaciones en cuanto a los valores de las sumas mensuales de las concentraciones diarias, durante el período común de estudio. En general puede observarse que son tres las épocas en las que suele presentarse este tipo: durante el verano, principalmente entre agosto y septiembre, en el otoño y en el invierno, siendo la primera de ellas constante en todos los años de estudio, mientras que las otras dos son más variables en cuanto a su aparición. Un comportamiento fenológico, en parte similar, encontraron Caramiello et al. (1989), teniendo en cuenta que su estudio fue realizado en la parte septentrional de Italia (Turin), 
encontrando dos picos máximos, uno en agosto, atribuido al polen de A. vulgaris y el otro en septiembre, correspondiente a $A$. annua y $A$. verlotiorum. Para Frenguelli et al. (1986) también el pico de agosto corresponde a $A$. vulgaris y el de septiembre-octubre a $A$. verlotiorum, en estudios realizados en Piceno y Perugia (Italia) y Leiden y Helmond (Holanda). En cambio para Munuera et al. (1996), en un estudio del Murcia (SE de España) el pico obtenido al final del verano corresponde a A. campestris y el máximo otoñal es atribuido principalmente a $A$. herba-alba y en parte a $A$. barrelieri.

A partir, pues, de su baja representación, sus variaciones interanuales y la similitud de comportamiento entre las tres estaciones, y teniendo en cuenta el hecho de que las dos especies citadas en la Región son raras, y sus floraciones se llevan a cabo, de acuerdo con Devesa (1995), en abril y mayo, para $A$. verlotiorum, y en junio y julio, para A. vulgaris, meses en los que no se registran concentraciones de este polen en ninguna de las tres estaciones, se puede suponer que su aparición se debe, fundamentalmente, al transporte de polen desde fuentes situadas fuera de la Región.

La gran cantidad de correlaciones significativas encontradas, al menos en el caso de Badajoz y Cáceres, cuando se enfrentan los datos de concentraciones totales mensuales al de las estaciones españolas estudiadas durante 1995, 1996 y 1997, revelan un gran sincronismo en los períodos de polinosis de este taxon dentro de la Península, hecho que podría estar explicado por el origen alóctono del polen de Artemisia en Extremadura. Ademñas, hay que tener en cuenta que al utilizar los valores de los meses de agosto a dciembre sólo aparecen correlaciones con las estaciones más próximas y que presenten datos con cantidades importantes.

En cuanto a los resultados obtenidos en Badajoz a partir de las correlaciones con los parámetros meteorológicos, algunos de ellos son obvios, así, las correlaciones significativas y positivas con las temperaturas medias y máximas, y negativas con la humedad, vienen a incidir sobre la aparición de este tipo en los meses de verano. Sin embargo, las correlaciones encontradas con los parámetros eólicos son algo más reveladoras, así, las correlaciones significativas y positivas con los períodos de viento procedente de los cuadrantes 1 y 2 , indica que la fuente de este polen está situada al Este del captador, lo cual está reafirmado por la correlación significativa y negativa encontrada con el cuadrante 3 . A pesar de que estos datos meteorológicos sólo corresponden a una estación hay que tener en cuenta que según Font (1983) durante el verano y el otoño son dominantes los vientos procedentes del Este en la mitad oriental de la península, lugar del supuesto origen del polen de Artemisia detectado en Badajoz.

En conclusión estimamos que el polen de Artemisia encontrado en Extremadura tiene su origen más probable en un transporte a distancia desde la parte oriental de la península ibérica. Fenómeno ya descrito por otros autores para granos de polen en particular como los de Betula (Hjelmroos, 1991, 1992) o Cannabis (Cabezudo et al., 1997); o granos de polen en general (Cambon et al., 1992; Franzén et al., 1992; Comtois, 1997), siendo en todos los casos el viento el responsable de transportar el polen incluso varios centenares de kilómetros desde su fuente.

\section{BIBLIOGRAFÍA}

AIRA, M. J., A. DOPAZO, M. V. JATO y F. J. RODRÍGUEZ - 1998a- Aerobiología en Galicia: Estación de Santiago de Compostela (1997). Rea 4: 107-110.

AIRA, M. J., A. DOPAZO y F. J. RODRÍGUEZ 1998b- Aerobiología en Galicia: Estación de Santiago de Compostela (1995-1996). Rea 3: 77-80. 
ALERGOLÓGICA -1995- Factores epidemiológicos, clínicos y socioeconómicos de las enfermedades alérgicas en España. Sociedad Española de Alergología e Inmunologia Clínica e Inmunología Abelló S.A.

BELMONTE, J., M. CAÑO y J. M. ROURE -1998aAerobiología en Cataluña: Estación de Lleida (1997). Rea 4: 87-90.

BELMONTE, J., M. CAÑO y J. M. ROURE - 1998bAerobiología en Cataluña: Estación de Manresa (1997). Rea 4: 79-82.

BELMONTE, J., A. GUÀRDIA y J. M. ROURE 1998c- Aerobiología en Cataluña: Estación de Girona (1997). Rea 4: 83-86.

BELMONTE, J., A. GUÀRDIA y J. M. ROURE 1998d- Aerobiología en Cataluña: Estación de Tarragona (1997). Rea 4: 91-94.

BELMONTE, J., M. VENDRELL y J. M. ROURE 1998e- Aerobiología en Cataluña: Estación de Barcelona (1995-1996). Rea 3: 53-56.

BELMONTE, J., M. VENDRELL y J. M. ROURE 1998f- Aerobiología en Cataluña: Estación de Barcelona (1997). Rea 4: 71-74.

BELMONTE, J., M. VENDRELL y J. M. ROURE 1998g- Aerobiología en Cataluña: Estación de Bellaterra (1995-1996). Rea 3: 57-60.

BELMONTE, J., M. VENDRELL y J. M. ROURE 1998h- Aerobiología en Cataluña: Estación de Bellaterra (1997). Rea 4: 75-78.

BERMEJO, D., A. M. GARCÍA, P. SANCHO y C. VALERO -1998a- Aerobiología en Aragón: Estación de Zaragoza (1995-1996). Rea 3: 45-48. BERMEJO, D., A. M. GARCÍA, P. SANCHO y C. VALERO -1998b- Aerobiología en Aragón: Estación de Zaragoza (1997). Rea 4: 53-56.

CABEZUDO, B., F. J. TORO, M. RECIO y M. M. TRIGO -1998- Aerobiología en Andalucía: Estación de Estepona (1995-1996). Rea 3: 37-40.

CAMBON, G., J. C. RITCHIE \& P. GUINET -1992Pollen marqueur de transports à longue distance dans l'atmosphère du sud de l'Ontario (Canada). Can. J. Bot. 70:2284-2293.

CABEZUDO, B., M. RECIO, J.M. SÁNCHEZLAUlhÉ, M. M. TRIGO, F. J. TORO \& F. POLVORINOS -1997- Atmospheric transportation of marihuana pollen from north Africa the southwest of Europe. Atmospheric Environment 31(20):3323-3328.

CANDAU, P., F. J. GONZÁlEZ MINERO, J.
MORALES y A. M. PÉREZ TELLO -1998aAerobiología en Andalucía: Estación de Sevilla I (1995-1996). Rea 3: 41-44.

CANDAU, P., C. TOMÁS, F. J. GONZÁLEZ MINERO, J. MORALES y A. M. PÉREZ TELLO -1998b- Aerobiología en Andalucía: Estación de Sevilla 1 (1997). Rea 4: 49-52.

CARAMIELLO, R., C. SINISCALCO \& V. POLINI -1989- Analyses aéropalynologiques, morphométriques et phénologiques d'Artemisia. Grana 28:105-113.

CARIÑANOS, P., C. GALÁN, P. ALCÁZAR y E. DOMÍNGUEZ -1998a- Aerobiología en Andalucía: Estación de Chirivel (1995-1996). Rea 3: 9-12.

CARIÑANOS, P., C. GALÁN, P. ALCÁZAR y E. DOMÍNGUEZ -1998b- Aerobiología en Andalucía: Estación de Chirivel (1997). Rea 4: 17-20.

COMTOIS, P. -1997- Pollen dispersal and long distance transport: the case of thermophilic pollen in subarctic Canada. Grana 13(1):37-42.

DE PABLOS, L., C. GALÁN y E. DOMÍNGUEZ 1998- Aerobiología en Castilla La Mancha: Estación de Ciudad Real (1997). Rea 4: 57-60.

DEVESA, J. A -1995-Vegetación y Flora de Extremadura. Universitas Editorial. Badajoz.

DÍAZ DE LA GUARDIA, C. y F. ALBA -1998aAerobiología en Andalucía: Estación de Granada (1995-1996). Rea 3: 21-24.

DÍAZ DE LA GUARDIA, C. y F. ALBA - 1998bAerobiología en Andalucía: Estación de Granada (1997). Rea 4: 29-32.

FERNÁNDEZ, D., R. M. VALENCIA, A. VEGA y F. GOZALO-REQUÉS -1998a- Aerobiología en Castilla y León: Estación de León (1997). Rea 4: 61-64.

FERNÁNDEZ, D., R. M. VALENCIA , A. VEGA y E. SAGÜÉS - 1998b- Aerobiología en Castilla y León: Estación de León (1995-1996). Rea 3: 49-52.

FRANZÉN, L. G., M. HJELMROOS, P. KÅLLBERG, E. BRORSTRÖM-LUNDÉN, S. JUNTTO \& A.-L- SAVOLAINEN -1994- The 'Yellow snow' episode of Northern Fennoscandia, march 1991- A case study of long-distance transport of soil, pollen and stable organic compounds. Atmosferic Environment 28(22):3587-3604. 
FRENGUELLI, G., F. TH. M. SPIEKSMA, B. ROMANO, A. H. NIKKELS, G. MINCIGRUCCI, W. DANKAART \& E. BRICCHI -1986- Comparative study of some airborne pollen concentration in The Netherlands and central Italy: Alnus, Poaceae, Artemisia; 1982-1985. Proc. 3 Internat. Conf Aerobiology, Switzerland

FONT, I. -1983-Climatología de España y Portugal. Instituto Nacional de Meteorología, Madrid.

GALÁN, C., P. CARIÑANOS, P. ALCÁZAR, F. INFANTE, A. CASTRO y E. DOMÍNGUEZ 1998a- Aerobiología en Andalucía: Estación de Córdoba (1995-1996). Rea 3: 13-16.

GALÁN, C., P. CARIÑANOS, P. ALCÁZAR, F. INFANTE, A. CASTRO y E. DOMÍNGUEZ 1998b- Aerobiología en Andalucía: Estación de Córdoba (1997). Rea 4: 21-24.

GONZÁlEZ, F. J., J. MORALES, P. CANDAU, C. TOMÁS y A. M. TELLO -1998a- Aerobiología en Andalucía: Estación de Huelva (1995-1996). Rea 3: 25-28.

GONZÁlEZ, F. J., J. MORALES, P. CANDAU, C. TOMÁS y A. M. TELLO -1998b- Aerobiología en Andalucía: Estación de Huelva (1997). Rea 4: 33-36.

GONZALO, M. A., I. SILVA, R. TORMO, A. F. MUÑOZ, J. M. GARCÍA y M. I. ALVARADO -1997- Aeropalinología del área de Badajoz. Revista Portuguesa de Imunoalergologia 5(2): 160-168.

GUTIÉRREZ, M. y P. NAVARRO -1998Aerobiología en Madrid: Estación Ciudad Universitaria (1995-1996). Rea 3: 85-88.

GUTIÉRREZ, M., G. VÁZQUEZ y P. CERVIGÓN -1998- Aerobiología en Madrid: Estación Ciudad Universitaria (1997). Rea 4: 119-122.

HJELMROOS, M. - 1991- Evidence of long-distance transport of Betula pollen. Grana 30:215-228.

HJELMROOS, M. -1992- Long-distance transport of Betula pollen grains and allergic symptoms. Aerobiologia 8(2):231-236.

IGLESIAS, I., J. MÉNDEZ y C. SEIJO -1998aAcrobiología en Galicia: Estación de Ourense (1995-1996). Rea 3: 73-76.

IGLESIAS, I., J. MÉNDEZ y C. SEIJO -1998bAerobiología en Galicia: Estación de Ourense (1997). Rea 4: 111-114.

MORENO, A., A. F. MUÑOZ, R. TORMO y I.
SILVA -1998a- Aerobiología en Extremadura: Estación de Mérida (1996). Rea 3: 65-68.

MORENO, A., A. F. MUÑOZ, R. TORMO y I. SILVA - 1998b- Aerobiología en Extremadura: Estación de Mérida (1997). Rea 4: 99-102.

MORENO-GRAU, S., B. ELVIRA-RENDUELES, J. M. ANGOSTO, J. BAYO, J. MORENO, J. BELCHÍ y J. MORENO-CLAVEL -1998aAerobiología en Murcia: Estación de Cartagena (1997). Rea 4: 123-126.

MORENO-GRAU, S., B. ELVIRA-RENDUELES, J. M. ANGOSTO, J. BAYO, J. MORENO y J. MORENO-CLAVEL - 1998b- Aerobiología en Murcia: Estación de Cartagena (1995-1996). Rea 3: 89-92.

MUNUERA, M., J. S. CARRIÓN \& J. GUERRA 1995- Approaches to airborne pollen in SE Spain. First survey in Murcia: one year of pollen monitoring (1993-94). Aerobiologia 11: 189-194.

MUNUERA, M., J. S. CARRIÓN \& J. GARCÍA 1996- Winter blooming of Artemisia. Two years survey in Murcia (Spain). In M. J. Aira, V. Jato, I. Iglesias \& C. Galán (eds.) 1st European Symposium on Aerobiology, Santiago de Compostela.

RECIO, M., M. M. TRIGO, F. J. TORO y B. CABEZUDO - 1998- Aerobiología en Andalucía: Estación de Málaga (1997). Rea 4: 41-44.

RODRÍGUEZ, F. J., M. R. DÍAZ y V. JATO 1998a- Aerobiología en Galicia: Estación de Vigo (1995-1996). Rea 3: 81-84.

RODRÍGUEZ, F. J., M. R. DÍAZ y V. JATO 1998b- Aerobiología en Galicia: Estación de Vigo (1997). Rea 4: 115-118.

RUIZ, L., C. DÍAZ DE LA GUARDIA y E. CANO -1998a- Aerobiología en Andalucía: Estación de Jaén (1995-1996). Rea 3: 29-32.

RUIZ, L., C. DÍAZ DE LA GUARDIA y E. CANO -1998b- Aerobiología en Andalucía: Estación de Jaén (1997). Rea 4: 37-40.

SILVA, I., A. F. MUÑOZ y R. TORMO -1998aAerobiología en Extremadura: Estación de Badajoz (1997). Rea 4: 95-98.

SILVA, I., A. F. MUÑOZ, R. TORMO y D. RECIO -1998b- Aerobiología en Extremadura: Estación de Badajoz (1995-1996). Rea 3: 61-64.

SPIEKSMA, F. TH. M. \& P. G. VON WAHL - 1991Allergenic significance of Artemisia (mugwort) 
pollen. En: G. D'Amato, F. Th. M. Spieksma \& S. Bonini (eds.) Allergenic pollen and pollinosis in Europe 121-124. Blackwell Scientific Publications. Oxford.

TAVIRA, J., R. TORMO, I. SILVA y A. F. MUÑOZ -1998a-Aerobiología en Extremadura: Estación de Cáceres (1996). Rea 3: 69-72.

TAVIRA, J., R. TORMO, I. SILVA y A. F. MUÑOZ -1998b- Aerobiología en Extremadura: Estación de Cáceres (1997). Rea 4: 103-106.

TORO, F. J., M. RECIO, B. CABEZUDO y M. M. TRIGO - 1998- Aerobiología en Andalucía: Estación de Estepona (1997). Rea 4: 45-48.

TRIGO, M. M., M. RECIO, F. J. TORO y B. CABEZUDO - 1998- Aerobiología en Andalucía: Estación de Málaga (1995-1996). Rea 3: 33-36.

TUTIN, T. G., K. PERSSON \& W. GUTERMANN -1976-Artemisia. En: T. G. Tutin et al. (eds.) Flora Europaea 4. Cambridge University Press. Cambridge.
VALENCIA, R. M., D. FERNÁNDEZ, A. VEGA, C. DÍAZ y J. MENCÍA -1998-Aerobiología en Castilla y León: Estación de Ponferrada (1997). Rea 4: 65-68.

Aceptado para su publicación en julio de 2000

Dirección de los autores. Escuela de Ingenierías Agrarias. Universidad de Extremadura. crta. Cáceres s/n. 06071 Badajoz (Inmaculada Silva, insilva @unex.es; Adolfo F. Muñoz, arodri@unex.es; Alfonsa Moreno), Facultad de Ciencias. Universidad de Extremadura. crta. Elvas s/n 06071 Badajoz (Rafael Tormo, ratormo@unex.es; Juana Tavira). 